\title{
The p.A897KfsX4 frameshift variation in desmocollin-2 is not a causative mutation in arrhythmogenic right ventricular cardiomyopathy
}

\author{
Marzia De Bortoli ${ }^{1}$, Giorgia Beffagna ${ }^{1}$, Barbara Bauce ${ }^{2}$, Alessandra Lorenzon ${ }^{1}$, Gessica Smaniotto ${ }^{1}$, \\ Ilaria Rigato ${ }^{2}$, Martina Calore ${ }^{1}$, Ilena EA Li Mura ${ }^{1}$, Cristina Basso ${ }^{3}$, Gaetano Thiene ${ }^{3}$, Gerolamo Lanfranchi ${ }^{1,4}$, \\ Gian Antonio Danieli ${ }^{1}$, Andrea Nava ${ }^{2}$ and Alessandra Rampazzo ${ }^{\star, 1}$
}

\begin{abstract}
Mutations in genes encoding desmosomal proteins have been reported to cause arrhythmogenic right ventricular cardiomyopathy/ dysplasia (ARVC/D), an autosomal-dominant disease characterised by progressive myocardial atrophy with fibro-fatty replacement. We screened 112 ARVC/D probands for mutations in desmocollin-2 (DSC2) gene and detected two different amino-acid substitutions (p.E102K, p.1345T) and a frameshift variation (p.A897KfsX4) in 7 (6.2\%) patients. DSC2a variant p.A897KfsX4, previously reported as a p.E896fsX900 mutation, was identified in five unrelated probands. Four of them were found to carry one or two mutations in different ARVC/D genes. Unexpectedly, p.A897KfsX4 variation was also found in 6 (1.5\%) out of 400 control chromosomes. In vitro functional studies showed that, unlike wild-type DSC2a, this C-terminal mutated protein was localised in the cytoplasm. p.A897KfsX4 variation affects the last five amino acids of the DSC2a isoform but not of DSC2b. In contrast with what we found in other human tissues, in the heart DSC2b is more expressed than DSC2a, suggesting that relative deficiency of DSC2a might be compensated by isoform b. In conclusion, DSC2 gene mutations are not frequently involved in ARVC/D. The p.A897KfsX4 variation, identified in several Italian healthy control subjects, which affects only one of the two DSC2 isoforms, may be considered a rare variant, though possibly affecting phenotypic expression of concomitant ARVC/D mutations.
\end{abstract}

European Journal of Human Genetics (2010) 18, 776-782; doi:10.1038/ejhg.2010.19; published online 3 March 2010

Keywords: sudden death; ARVC/D; desmosome; DSC2; polymorphism

\section{INTRODUCTION}

Arrhythmogenic right ventricular cardiomyopathy/dysplasia (ARVC/D) (MIM \#107970) is an inherited disorder characterised by progressive fibro-fatty replacement of the right ventricular myocardium. ${ }^{1}$ Clinical manifestations occur most often between the second and fourth decades of life and are characterised by ventricular arrhythmias, heart failure and sudden death. The disease usually shows autosomaldominant inheritance with reduced penetrance, ${ }^{2}$ although autosomalrecessive transmission has also been reported in Naxos Syndrome. ${ }^{3}$

Eight genes have been detected so far as being independently involved in the pathogenesis of the disease. Five of them encode major desmosomal proteins: plakoglobin $\left(\mathrm{MIM}{ }^{\star} 173325\right),{ }^{3,4}$ desmoplakin $\left(\right.$ MIM $\left.{ }^{\star} 125647\right),{ }^{5}$ plakophilin-2 $\left(\right.$ MIM $\left.^{\star} 602861\right),{ }^{6}$ desmoglein-2 $\left(\mathrm{MIM}{ }^{\star} 125671\right)^{7}$ and desmocollin-2 (MIM $\left.{ }^{\star} 125645\right), 8,9$ The involvement of such genes led to the current idea that ARVC/D is a disorder caused mainly by defects in cell-cell adhesion.

Other three nondesmosomal genes have been associated with ARVC/D: cardiac ryanodine receptor 2 (RYR2; MIM 180902), ${ }^{10}$ transforming growth factor beta-3 (TGF $\beta 3$; MIM 190230) ${ }^{11}$ and transmembrane protein 43 (TMEM43; MIM 612048). ${ }^{12}$

To date, five different DSC2 mutations have been reported: two frameshift mutations p.M477fsX480 and p. E896fsX900, ${ }^{8}$ a heterozygous splice acceptor site mutation c.631-2A $>\mathrm{G}^{9}$ and two missense mutations p.E102K and p.I345T. ${ }^{13}$ In vitro functional studies showed that the two point mutations affect the intracellular localisation of DSC2a, thus suggesting a potential pathogenic effect. ${ }^{13}$ More recently, a homozygous mutation p.S614fsX625 in DSC2 gene associated with autosomal-recessive ARVC/D, mild palmoplantar keratoderma and woolly hair has been described. ${ }^{14}$

In this study, we investigated the frequency of DSC2 mutations in an unselected Italian ARVC/D patient cohort. In five probands, we have identified the frameshift p.A897KfsX4 variation (previously reported as p.E896fs $\mathrm{X} 900^{8}$ ), which was also detected among Italian healthy control subjects. On the basis of genetic and functional data, we discuss here the pathogenic role of the p.A897KfsX4 variation.

\section{MATERIALS AND METHODS}

Mutation screening

A total of 112 ARVC/D unrelated index cases were screened for DSC2 mutations by denaturing high-performance liquid chromatography and direct sequencing. This patient group includes 54 subjects already reported in a previous study. ${ }^{13}$ The coding region of DSP, PKP2 and DSG2 genes was screened for mutations in DSC2 mutation carriers.

A control group of 200 healthy and unrelated Italian subjects (400 alleles) was used to exclude that identified mutations could be DNA polymorphisms. All controls were matched to the probands by ancestry and underwent ordinary clinical investigations. 
Mutation screening was performed in all available family members of index cases in which a DSC2 mutation was detected.

Comparison of p.A897KfsX4 variant in patients and control subjects was assessed by one-tail Fisher's exact test. A $P$-value $<0.05$ was considered statistically significant.

\section{Clinical study}

Each patient underwent physical examination and family history, 12-lead electrocardiography (ECG), signal-averaged ECG, 24-h Holter ECG and twodimensional echocardiography. A clinical diagnosis of ARVC/D was based on major and minor criteria, established by the European Society of Cardiology/ International Society and Federation of Cardiology Task Force. ${ }^{15}$ Informed consent for clinical investigations and blood sampling for DNA analysis was obtained from all participating individuals, according to the pertinent Italian legislation and in compliance with the Helsinki declaration.

\section{Haplotype analysis}

DNA samples of all frameshift variation carriers were assessed for a common haplotype using the following microsatellite markers: D18S847, D18SH3, D18SH4 and D18S36. Markers D18SH3 and D18SH4 are new polymorphic tri- and dinucleotide repeats that we identified starting at positions 26755775 and 26908386, respectively, on chromosome 18q12.1 (Human Genome Browser, http://genome.ucsc.edu/). They were amplified using the primers D18SH3F 5'-GTGGTGGGCATCTGTAATCC-3', D18SH3R 5'-GGTGCCTGCGTTTAGTA T-3', D18SH4F 5'-CTCCCTTATGACCCAGGAAA-3' and D18SH4R 5'-ACCAT GTGGGAAACACCAAT- $3^{\prime}$ under standard PCR conditions. D18SH4 is within the DSC2 intron 12, whereas the other three markers are in close proximity to the DSC2 locus. Forward primers were fluorescently labelled. In particular, D18S847 and D18SH3 were labelled with TAMRA, D18SH4 with HEX and D18S36 with FAM. Amplification products were pooled into a post-amplification panel, mixed with GeneScan ROX400 size standard (Applied Biosystems, Foster City, CA, USA). Capillary electrophoresis was carried out on an ABI PRISM 3730XL DNA sequencer (Applied Biosystems) and Genotyper V3.5 analysis software (Applied Biosystems) was used to analyse each amplicon.

\section{Expression pattern of $D S C 2$ isoforms}

Expression of DSC2a and DSC2b isoforms in different human tissues was examined by PCR amplification of cDNAs including the heart, brain, placenta, lung, skeletal muscle, liver, kidney and pancreas (Multiple Tissue cDNA panel I, Clontech, Palo Alto, CA, USA). Amplification was performed by using primers previously reported. ${ }^{16}$ The splice form ' $\mathrm{b}$ ' resulted in a PCR fragment of $500 \mathrm{bp}$, whereas splice form 'a' produced a $454 \mathrm{bp}$ fragment.

\section{Site-directed mutagenesis}

PCR-based site-directed mutagenesis was performed on wild-type DSC2apcDNA3.1/CT-GFP, already available to the study, ${ }^{13}$ to obtain three new constructs. Each construct differs from the others for a variation introduced in the human DSC2-coding region: an insertion c.2687_2688insGA leading to the frameshift variation p.A897KfsX4, and two nucleotide substitutions c.536A > G (p.D179G) and c.2393G >A (p.R798Q), which happened to be common polymorphisms. The following mutagenic primers were used: DSC2D179G: 5'-TCCATAAGAGGTCCTGGAGTTGGCCAAGAACCCGGAAT TTATTTT-3'; DSC2R798Q: 5'-CACCAGACCTCGḠAATCCTGCCAGGGGG CTGCCACCATCACACC-3'; DSC2insGA: $5^{\prime}$-CAAATTTGGACACTAG $\bar{G}$ CAGAG AAGCATGCATGAAGAGACAAG-3' and DSC2frameGA: 5'-CACTAGCA $\overline{G A G}$ AAGCATGCACAAGGGCAATTCTGCAGATA-3'. Two steps of mutagenesis were necessary to obtain a construct coding for p.A897KfsX4 variation, the first to introduce the GA insertion and the second to remove the premature stop codon and the following 5 nucleotides to get the right frame with GFP sequence. Thus, the mutant construct does not contain additional amino acids compared with the wild type. The coding regions of each mutant construct were fully sequenced.

\section{Cell culture and transfection}

HL-1 cells, kindly provided by Dr WC Claycomb (New Orleans), were used and maintained as previously reported. ${ }^{17}$ Cells were cultured at $37^{\circ} \mathrm{C}$ in $5 \% \mathrm{CO}_{2}$.

Constructs corresponding to wild type or to a variant of human DSC2a were transiently transfected in HL-1 cells at a confluence of 70-80\%, using Effectene reagent (Qiagen, Hilden, Germany) according to the manufacturer's instructions. Transfected cells were incubated for $48 \mathrm{~h}$ to allow protein expression and desmosome formation.

\section{Immunostaining and confocal imaging}

HL-1 cells were fixed with cold methanol/acetone (1:1) and immunostained for endogenous desmoglein with the murine mAb (clone DG3.10) as previously described in detail. ${ }^{13}$ Images were acquired with a Radiance 2000 confocal microscope (BioRad, Hercules, CA, USA) with a $\times 60$ oil objective.

\section{RESULTS}

\section{Mutation screening}

DSC2 mutation screening was performed in a series of 112 consecutive unrelated index cases. Two different amino-acid substitutions (p.E102K, p.I345T) and a frameshift (p.A897KfsX4) have been detected in seven $(6.2 \%)$ patients (Figure 1a). We also identified two polymorphisms (c.536A $>\mathrm{G}$ and c.2393G $>$ A, allele frequency 2.7 and $4.7 \%$, respectively) in exon 5 and in exon 15 , resulting in predicted p.D179G (novel) and p.R798Q (rs61731921) amino-acid substitutions.

The two missense mutations p.E102K and p.I345T, previously reported by our group, ${ }^{13}$ affect the normal localisation of mutant proteins in cultured cardiomyocytes. The frameshift variation p.A897KfsX4, primarily reported as p.E896fsX900 by Syrris et al, ${ }^{8}$ causes a premature termination of the protein. Only the last five amino acids of DSC2a were altered: three were changed and the last two were lost (Figure 1b). The five involved amino-acid residues are

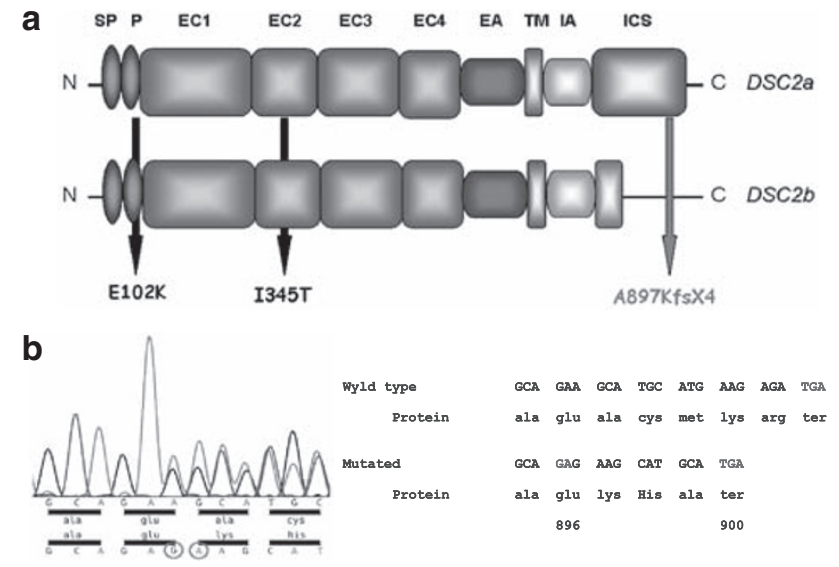

C Homo_sapiens Macaca mulatta Pan troglodytes Bos taurus Rattus_norvegicus Canis familiaris Equus_caballus

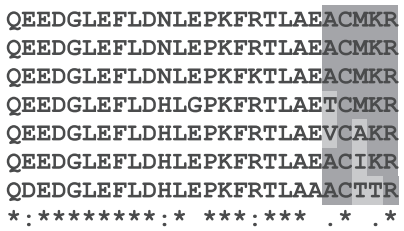

Figure 1 (a) p.E102K and p.I345T mutations (black arrows) and p.A897KfsX4 variation (red arrow) identified in the DSC2 gene. p.A897KfsX4 variation affects only the DSC2a isoform. (b) Changes introduced in the amino-acid sequence of DSC2a, caused by the insertion c.2687_2688insGA in the DSC2 gene. (c) The last five amino acids of DSC2a (involved by p.A897KfsX3 variation) show relative variance among the species: Homo sapiens (NP_077740), predicted Macaca mulatta (XP_001102096.1), predicted Pan troglodytes (XP_512077.2), predicted Bos taurus (XP_615164.3), Rattus norvegicus (NP_001028860.1), predicted Canis familiaris (XP_866917.1), predicted and Equus caballus (XP_001496356.1). The colour reproduction of the figure is available on the html full text version of the paper. 
Table 1 A897KfsX4 variation carriers

\begin{tabular}{|c|c|c|c|}
\hline Proband & Gene & Nucleotide change & Amino-acid change \\
\hline 1 & DSC2 & c. 2687 2688insGA & p.A897KfsX4 \\
\hline \multirow[t]{2}{*}{2} & DSC2 & c.2687_2688insGA & p.A897KfsX4 \\
\hline & DSG2 & c. $260 A>G$ & p.Y87C \\
\hline \multirow[t]{3}{*}{3} & DSC2 & c.2687_2688insGA & p.A897KfsX4 \\
\hline & DSG2 & c. $1912 \mathrm{G}>\mathrm{A}$ & p.G638R ${ }^{a}$ \\
\hline & DSP & c. $1124 \mathrm{~A}>\mathrm{T}$ & p.N3751 \\
\hline \multirow[t]{2}{*}{4} & DSC2 & c.2687_2688insGA & p.A897KfsX4 \\
\hline & PKP2 & c.1211_1212insT & p.V406SfsX3 \\
\hline \multirow[t]{3}{*}{5} & DSC2 & c.2687_2688insGA & p.A897KfsX4 \\
\hline & DSP & c. $1124 \mathrm{~A}>\mathrm{T}$ & p.N375l ${ }^{a}$ \\
\hline & PKP2 & c. $1655 \mathrm{~T}>\mathrm{G}$ & p.F552C \\
\hline
\end{tabular}

aindicates novel mutations.

less conserved among mammals, in contrast with the high conservation of the upstream region (Figure 1c). DSC2b is not affected by the p.A897KfsX4 variation (Figure 1a), as it has a shortened C-terminal domain caused by a stop codon in the additional exon 16 .

Five unrelated ARVC/D index cases (allele frequency 2.2\%) were found to carry the p.A897KfsX4 variation, as well as 6 out of 200 control subjects (allele frequency 1.5\%).

A $0.11 P$-value, falling above the 0.05 significance threshold, indicates that there was no evidence of overrepresentation of the p.A897KfsX4 variant in patients compared with control subjects.

Mutations in other ARVC/D genes were identified in four of these five patients (Table 1). Proband 1 was found to carry the p.A897KfsX4 mutation and the previously reported PKP2 p.E58D common polymorphism. ${ }^{18}$ The allele frequency of the p.E58D variant in our control population was $0.9 \%$, in contrast with the reported $5 \%$ in the Finnish population representing a genetic isolate. ${ }^{18}$

The additional detected mutations DSG2 p.Y87C and PKP2 p.V406SfsX3 have been previously reported as disease causing. ${ }^{19,20}$ None of the novel identified mutations (DSG2 p.G638R; DSP p.N375I; PKP2 p.F552C) were observed in 400 control chromosomes and occurred in residues that are highly conserved among species (data not shown). Moreover, they all lead to a change in biochemical properties of the amino acid involved.

In proband 4 , it has also been identified a nucleotide substitution c. $45 \mathrm{C}>\mathrm{T}$ in the PKP2 gene, which causes a synonymous variation p.T15T. A total of 220 control alleles are negatives for this variation. This nucleotide substitution might be a rare polymorphism or it might activate a cryptic splice site and produce an aberrant transcript.

\section{Clinical findings}

Proband 1 was a 60-year-old woman who was diagnosed as having ARVC/D due to ECG abnormalities. A two-dimensional echocardiogram revealed a severe dilation of the right ventricle with depressed ventricular function. Moreover, frequent premature ventricular contractions with left bundle-branch block morphology were present at 24-h Holter ECG. The genetic analysis extended to her son revealed that he carries the DSC2 frameshift variation p.A897KfsX4 and PKP2 polymorphism p.E58D as well. Clinical evaluation showed the presence of some criteria also in the son, even if he did not fulfil the diagnosis (Table 2).

Proband 2 was diagnosed at the age of 45 with palpitations. The 24-h Holter ECG showed frequent episodes of non-sustained ventricular arrhythmias and imaging techniques showed a severe right ventricular dilation/dysfunction. He was found to be a double heterozygote for the DSC2 p.A897KfsX4 variation and the DSG2 p.Y87C mutation. His daughter, clinically unaffected, is negative for both (Table 2).

Proband 3 experienced a sustained VT episode at the age of 38 years. Clinical and instrumental examination revealed at that time right ventricular abnormalities that became more evident during follow-up. She also carried a missense mutation in the DSP gene (p.N375I) and in the DSG2 gene (p.G638R). The genetic study was extended to additional family members, and two subjects were double heterozygotes for the DSG2 mutation and DSC2 variation and one subject carried the DSG2 mutation (Figure 2). One of the double heterozygotes (subject 3.(II,7)) carrying the DSG2 p.G638R and DSC2 p.A897KfsX4 fulfilled the ARVC/D diagnostic criteria, whereas the other family members were clinically unaffected (Table 2).

Proband 4 was a female patient who underwent cardiac evaluation due to several presyncopal episodes. The ECG showed negative $\mathrm{T}$ waves in V1-V4 and in inferior leads. Late potentials were present at 40-80 Hz. At two-dimensional echocardiogram, the right ventricle was mildly dilated with the presence of kinetic alterations; the 24-Holter ECG documented the presence of frequent premature ventricular contractions with left bundle-branch block morphology.

Cardiac magnetic resonance confirmed the presence of mild right ventricular dilatation and kinetic alterations, localised on the subtricuspid region and on the apex. The left ventricle was not involved. She was also found to carry the PKP2 p.V406SfsX3 mutation and the PKP2 p.T15T synonymous variation. The genetic analysis extended to her daughter revealed that she carries only the p.T15T variation in the presence of negative clinical and instrumental findings.

Proband 5 underwent cardiac evaluation at the age of 45 years, because of the presence of premature ventricular contractions detected during a pre-operatory ECG that also showed intraventricular conduction delay and negative T waves in V4-V6. Signal-averaged ECG documented the presence of late potentials at all filter settings. The two-dimensional echocardiogram showed biventricular dimensional and kinetic abnormalities. At the age of 49 , he received an implantable cardioverter defibrillator because of recurrent VT episodes. He was found to carry additional mutations in the other two ARVC/D genes: p.N375I in DSP and p.F552C in PKP2. His brother carries only the DSP mutation. The clinical and instrumental examination showed the presence of some ECG criteria, even if he did not fulfil the diagnosis (Table 2).

\section{Haplotype analysis}

In contrast with other reported DSC2 mutations, ${ }^{8,9,13}$ p.A897KfsX4 is the only one identified in more Italian and English ARVC/D patients. Haplotype analysis was performed using four polymorphic microsatellites, one intragenic (D18SH4) and three in close proximity to the DSC2 gene (D18S847, D18SH3 and D18S36). Different haplotypes segregated with the p.A897KfsX4 variation in each of the five Italian patients showing the absence of a founder effect for this variation (data not shown). This result confirms data reported by Syrris et al ${ }^{8}$ for their three families, suggesting that p.A897KfsX4 is a recurrent variation.

\section{Expression pattern of DSC2 isoforms}

Almost all analysed human cDNA samples (heart, pancreas, lung, placenta, brain, skeletal muscle, liver and kidney) showed expression of both DSC2 splice forms (Figure 3). It is interesting to note that all of them showed a different expression level of the two isoforms. In particular, in contrast with what we found in the other tissues, in the heart, isoform $b$ is more expressed than isoform a. 


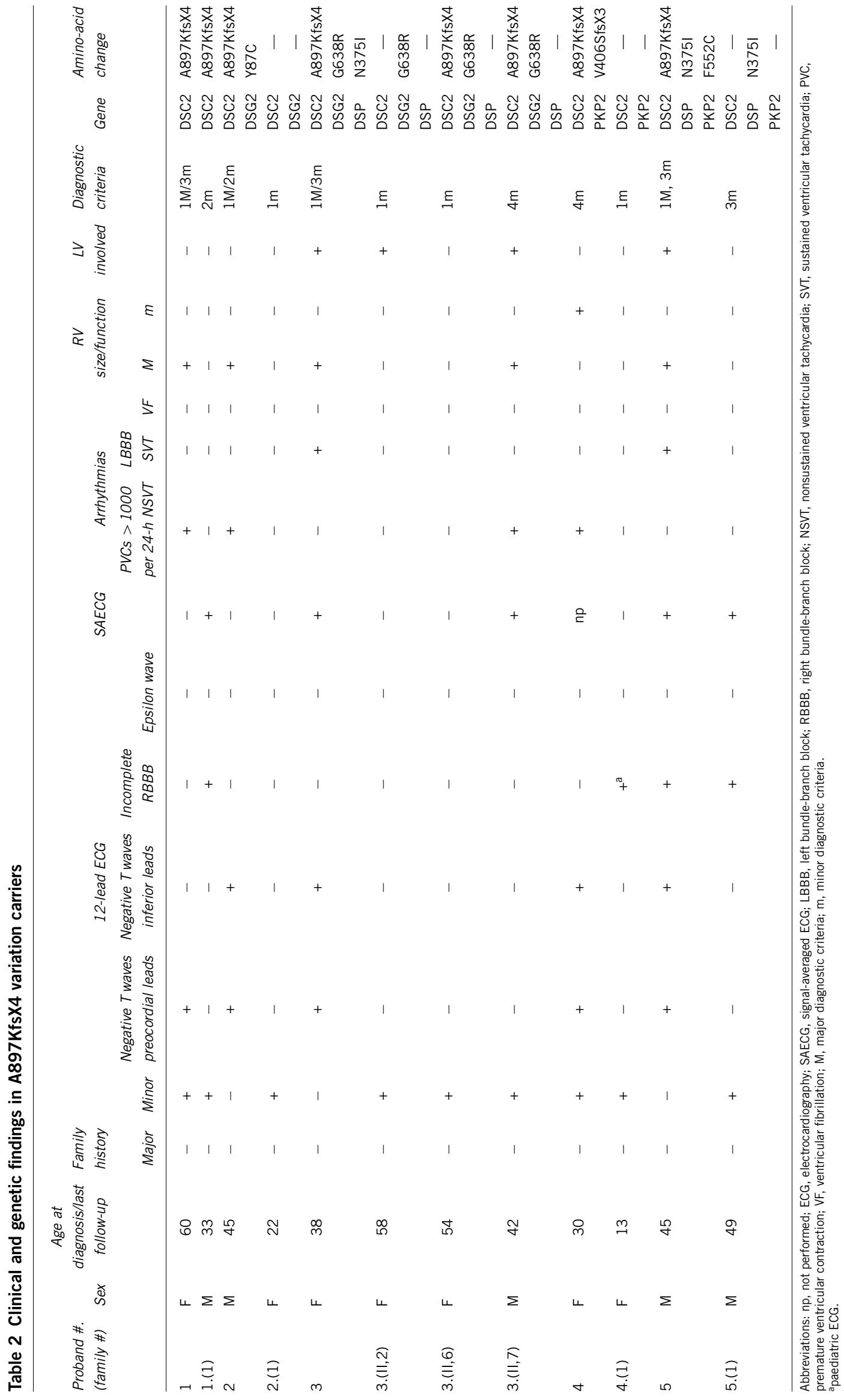




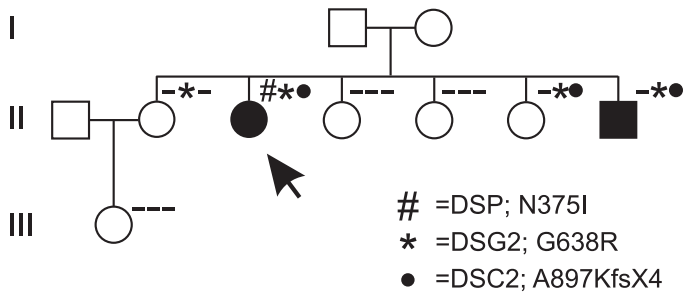

Figure 2 Pedigree of proband 3 (marked by an arrow). Filled-in symbols indicate clinically affected family members and white symbols indicate unaffected individuals. Absence (-) and presence of (\#) DSP, (*) DSG2 mutations and $(\bullet) D S C 2$ variation are indicated.

The PCR products for heart tissue were verified and confirmed by sequencing (data not shown).

\section{Functional analysis of C-terminal mutant DSC2a (p.A897KfsX4)}

Site-directed mutagenesis was performed on wild-type construct encoding for a human DSC2a-GFP fusion protein to study the functional effect of the p.A897KfsX4 variation and of two DSC2 polymorphisms, p.D179G and p.R798Q, located in the $\mathrm{N}$ - and C-terminal domains of the protein, respectively.

Constructs were transfected in the desmosome-forming cell line HL-1 having a differentiated cardiomyocyte phenotype and contractile activity in vitro. The GFP signal revealed a predominant localisation at the plasma membrane of wild type (Figure $4 \mathrm{a}$ ) and proteins carrying p.D179G and p.R798Q polymorphisms (Figures $4 \mathrm{~b}$ and c), displaying colocalisation with endogenous dsg at the cell membrane to indicate well-assembled desmosomes (Figure $4 \mathrm{a}^{\prime \prime}-\mathrm{c}^{\prime \prime}$ ).

By contrast, protein-carrying frameshift variation p.A897KfsX4 was detected in the cytoplasm (Figure 4d), losing the proper desmosomal localisation along cell boundaries. However, endogenous dsg is normally well distributed (Figure $4 \mathrm{~d}^{\prime}$ ).

\section{DISCUSSION}

DSC2 was the most recent major component of the cardiac desmosome to be implicated in ARVC/D. DSC2 gene encodes one of desmosomal cadherins, single-pass transmembrane glycoproteins able to mediate $\mathrm{Ca}^{2+}$-dependent cell-cell adhesion, by interacting laterally and transcellularly with each other and by recruiting cytoplasmic plaque proteins that facilitate attachment of intermediate filaments. $^{21}$

We have identified two different DSC2 mutations and one DSC2 variation in 7 out of 112 unrelated ARVC/D index cases, two already described in our previous report ${ }^{13}$ and p.A897KfsX4 originally referred to as a causative mutation. ${ }^{8}$ The frameshift variation was identified in five independent patients, four of whom are carriers for one or two mutations in known ARVC/D genes. Surprisingly, we found the same variation in several Italian controls with no significant difference in the distribution of the variant between the patients and control group.

By contrast, in the original paper reporting the identification of the p.A897KfsX4 variation, three probands carried only this frameshift variation, which was never identified in 200 control subjects. ${ }^{8}$

In contrast with other reported DSC2 mutations, p.A897KfsX4 is the only one identified in several Italian and English ARVC/D patients. Haplotype analysis excludes a founder effect in our patients as well as in English cases and confirms that p.A897KfsX4 is a recurrent variation.

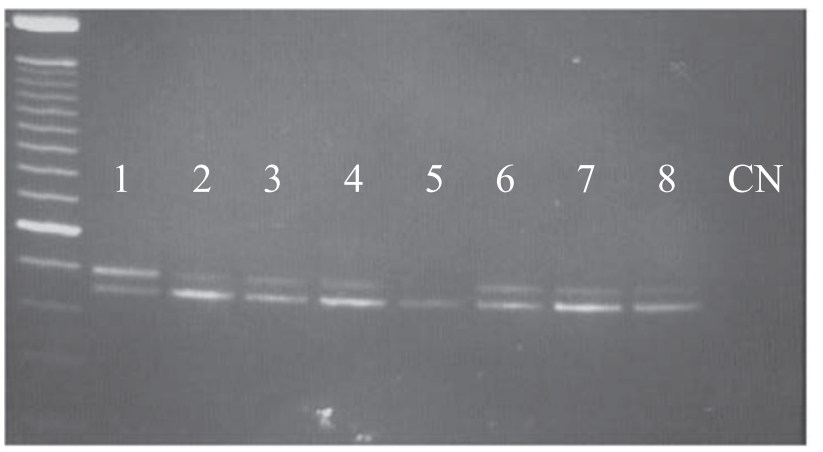

Figure 3 Expression pattern of $D S C 2$ splice forms. Double band represents isoform b $(500 \mathrm{bp})$ and isoform a $(454 \mathrm{bp})$. Results shown are from the heart (lane 1), pancreas (lane 2), lung (lane 3), placenta (lane 4), brain (lane 5), skeletal muscle (lane 6), liver (lane 7) and kidney (lane 8).

This variant occurs in exon 17 that encodes for the intracellular cadherin-like sequence (ICS) domain only in the splice variant 'a' of the DSC2 gene, whereas the splice variant ' $\mathrm{b}$ ' has a different C-terminal domain. ICS domain provides binding sites for other desmosomal constituents such as plakoglobin, plakophilin and desmoplakin. ${ }^{22-24}$ It has been shown that the latest 37 amino acid in Dscla are fundamental for plakoglobin binding in human epithelial A-431 cells. ${ }^{23}$ On the other hand, in vitro functional studies on HL-1 cells have shown that DSC2a-GFP-A897KfsX4 protein localises in the cytoplasm, in contrast with wild-type protein and DSC2a variants carrying the polymorphism p.D179G or p.R798Q, which correctly colocalise at the cell membrane with endogenous desmoglein.

However, it is important to notice that the exon 17 is untranslated in the DSC2b isoform. Therefore, the insertion would affect only DSC2a, leaving isoform $b$ fully functional and possibly able to compensate in cardiac myocytes the relative deficiency of the DSC2a isoform. Furthermore, in contrast with what we found in the other human tissues, in the heart, the DSC2b isoform is more expressed than DSC2a, supporting the hypothesis of a possible compensation by the isoform $b$.

Little is known about human DSC2b and in general about all Dscb isoforms; until now, a specific desmosomal interaction with PKP3 has been shown only for Dsc3b. ${ }^{25}$ Moreover, PKP1, for which strong in vitro association with the 'a' form of Dscl has been reported, ${ }^{24}$ was also found to overlap with the ' $b$ ' form. ${ }^{26}$ The possibility that Dscb isoforms contain a PKP-binding domain should be investigated further.

Four out of five patients carrying the p.A897KfsX4 variation also had one or two additional ARVC/D mutations. On the other hand, proband 1 carrying only the p.A897KfsX4 variation was also found to carry a reported polymorphism (PKP2 p.E58D) whose pathogenic role, if any, remains to be investigated. ${ }^{18}$ Moreover, we cannot exclude that this proband could have a large insertion, deletion or mutation in the non-coding regions of ARVC/D genes or also a mutation in unknown ARVC/D genes.

It may be hypothesised that the p.A897KfsX4 variation could act in the presence of other ARVC/D mutations as a factor able to modify their pathogenic effect. All probands showed moderate to severe forms of the disease, with biventricular involvement in some cases. However, in our study group, genotype-phenotype data could not give a clear indication of the role of this variant in the disease phenotype. Moreover, there is no different proportion 

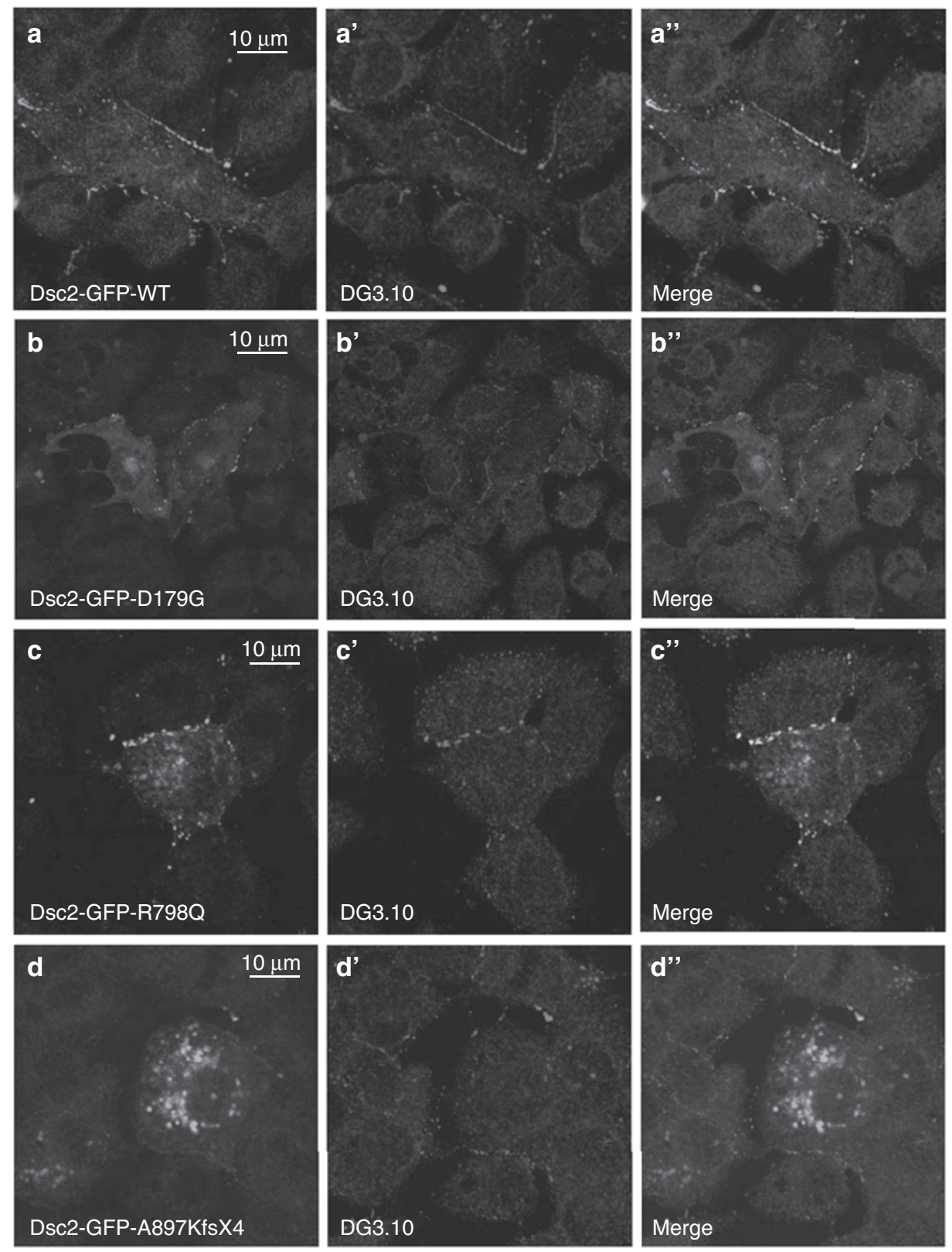

Figure 4 Functional studies in HL-1 cells. DSC2a-GFP-WT, DSC2a-GFP-D179G and DSC2a-GFP-R798Q were localised at the cell membrane between two $\mathrm{HL}-1$ cells $(\mathbf{a}-\mathbf{c})$, whereas DSC2a-GFP-A897KfsX4 was detected in the cytoplasm (d). Immunostaining with monoclonal desmoglein antibody DG3.10 $\left(a^{\prime}-d^{\prime}\right)$ showed both the presence of well-assembled desmosomes (yellow dots in $\mathbf{a}^{\prime \prime}-\mathbf{c}^{\prime \prime}$ ) and no colocalisation between endogenous dsg and DSC2a-GFPA897 Kfs X4 $\left(\mathbf{d}^{\prime \prime}\right)$. The colour reproduction of the figure is available on the $\mathrm{html}$ full text version of the paper.

of the variant in the controls and affected subjects to provide evidence of an association between the disease and the variant. Assessment of the exact significance of the p.A897KfsX4 variation requires further studies in additional families carrying mutations in other ARVC/D genes.

Until now, few DSC2 gene mutations were reported to cause ARVC/D. Among them, the p.A897KfsX4 variation, identified in several Italian healthy control subjects and altering only one of the two DSC2 isoforms, could be considered a rare polymorphism that may affect the phenotypic expression of concomitant ARVC/D mutations.

Further clarification of the co-occurrence of mutations and rare polymorphisms in different desmosomal proteins could be an important aspect in accounting for the known inter- and intrafamilial variable phenotypic expression.

\section{CONFLICT OF INTEREST}

The authors declare no conflict of interest.

\section{ACKNOWLEDGEMENTS}

This study was supported by Telethon, Rome (GGP07220); the Fifth Framework Programme European Commission (QLG1-CT-2000-01091); the Ministry of Health, MIUR (2006061007_002); and the Fondazione Cassa di Risparmio, Padova e Rovigo. The authors are deeply indebted to Paola Marcon for her help in collecting ARVC/D families. 
1 Thiene G, Nava A, Corrado D, Rossi L, Pennelli N: Right ventricular cardiomyopathy and sudden death in young people. N Engl J Med 1988; 318: 129-133.

2 Nava A, Thiene G, Canciani B et al: Familial occurrence of right ventricular dysplasia: a study involving nine families. J Am Coll Cardiol 1988; 12: 1222-1228.

3 McKoy G, Protonotarios N, Crosby A et al: Identification of a deletion in plakoglobin in arrhythmogenic right ventricular cardiomyopathy with palmoplantar keratoderma and woolly hair (Naxos disease). Lancet 2000; 355: 2119-2124.

4 Asimaki A, Syrris P, Wichter T, Matthias P, Saffitz JE, McKenna WJ: A novel dominant mutation in plakoglobin causes arrhythmogenic right ventricular cardiomyopathy. Am J Hum Genet 2007; 81: 964-973.

5 Rampazzo A, Nava A, Malacrida S et al: Mutation in human desmoplakin domain binding to plakoglobin causes a dominant form of arrhythmogenic right ventricular cardiomyopathy. Am J Hum Genet 2002; 71: 1200-1206.

6 Gerull B, Heuser A, Wichter T et al: Mutations in the desmosomal protein plakophilin-2 are common in arrhythmogenic right ventricular cardiomyopathy. Nat Genet 2004; 36: 1162-1164.

7 Pilichou K, Nava A, Basso C et al: Mutations in desmoglein-2 gene are associated with arrhythmogenic right ventricular cardiomyopathy. Circulation 2006; 113: 1171-1179.

8 Syrris P, Ward D, Evans A et al: Arrhythmogenic right ventricular dysplasia/cardiomyopathy associated with mutations in the desmosomal gene desmocollin-2. Am J Hum Genet 2006; 79: 978-984.

9 Heuser A, Plovie ER, Ellinor PT et al: Mutant desmocollin-2 causes arrhythmogenic right ventricular cardiomyopathy. Am J Hum Genet 2006; 79: 1081-1088.

10 Tiso N, Stephan DA, Nava A et al: Identification of mutations in the cardiac ryanodine receptor gene in families affected with arrhythmogenic right ventricular cardiomyopathy type 2 (ARVD2). Hum Mol Genet 2001; 10: 189-194.

11 Beffagna G, Occhi G, Nava A et al: Regulatory mutations in transforming growth factor-beta 3 gene cause arrhythmogenic right ventricular cardiomyopathy type 1 . Cardiovasc Res 2005; 65: 366-373.

12 Merner ND, Hodgkinson KA, Haywood AF et al: Arrhythmogenic right ventricular cardiomyopathy type 5 is a fully penetrant, lethal arrhythmic disorder caused by a missense mutation in the TMEM43 gene. Am J Hum Genet 2008; 82: 809-821.

13 Beffagna G, De Bortoli M, Nava A et al: Missense mutations in desmocollin-2 $\mathrm{N}$-terminus, associated with arrhythmogenic right ventricular cardiomyopathy, affect intracellular localization of desmocollin-2 in vitro. BMC Med Genet 2007; 8: 65-75.
14 Simpson MA, Mansour S, Ahnood D et al: Homozygous mutation of desmocollin-2 in arrhythmogenic right ventricular cardiomyopathy with mild palmoplantar keratoderma and woolly hair. Cardiology 2008; 113: 28-34.

15 McKenna WJ, Thiene G, Nava A et al: Diagnosis of arrhythmogenic right ventricular dysplasia/cardiomyopathy: Task Force of the Working Group Myocardial and Pericardial Disease of the European Society of Cardiology and of the Scientific Council on Cardiomyopathies of the International Society and Federation of Cardiology. Br Heart J 1994; 71: 215-218.

16 Nuber UA, Schafer S, Schmidt A, Koch PJ, Franke WW: The widespread human desmocollin Dsc2 and tissue-specific patterns of synthesis of various desmocollin subtypes. Eur J Cell Biol 1995; 66: 69-74.

17 Claycomb WC, Lanson NA, Stallworth BS et al: HL-1 cells: a cardiac muscle cell line that contracts and retains phenotypic characteristics of the adult cardiomyocyte. Proc Natl Acad Sci USA 1998; 95: 2979-2984.

18 Lahtinen AM, Lehtonen A, Kaartinen $\mathrm{M}$ et al: Plakophilin-2 missense mutations in arrhythmogenic right ventricular cardiomyopathy. Int J Cardiol 2008; 126: 92-100.

19 Pilichou K, Nava A, Basso C et al: Mutations in desmoglein-2 gene are associated with arrhythmogenic right ventricular cardiomyopathy. Circulation 2006; 113: 1171-1179.

20 van Tintelen JP, Entius MM, Bhuiyan ZA et al: Plakophilin-2 mutations are the major determinant of familial arrhythmogenic right ventricular dysplasia/cardiomyopathy. Circulation 2006; 113: 1650-1658

21 Yin T, Green KJ: Regulation of desmosome assembly and adhesion. Semin Cell Dev Biol 2004; 15: 665-677.

22 Troyanovsky SM, Eshkind LG, Troyanovsky RB, Leube RE, Franke WW: Contributions of cytoplasmic domains of desmosomal cadherins to desmosome assembly and intermediate filament anchorage. Cell 1993; 72: 561-574.

23 Troyanovsky SM, Troyanovsky RB, Eshkind LG, Leube RE, Franke WW: Identification of amino acid sequence motifs in desmocollin, a desmosomal glycoprotein, that are required for plakoglobin binding and plaque formation. Proc Natl Acad Sci USA 1994; 91: 10790-10794.

24 Smith EA, Fuchs E: Defining the interactions between intermediate filaments and desmosomes. J Cell Biol 1998; 141: 1229-1241.

25 Bonné S, Gilbert B, Hatzfeld M, Chen X, Green KJ, van Roy F: Defining desmosomal plakophilin-3 interactions. J Cell Biol 2003; 161: 403-416.

26 North AJ, Bardsley WG, Hyam J et al: Molecular map of the desmosomal plaque. J Cell Sci 1999; 112: 4325-4336. 